\title{
Cognitive Performance Degradation in High School Students as the Response to the Psychophysiological Changes
}

\author{
Oleksandr Burov ${ }^{1(\bowtie)}$, Evgeniy Lavrov ${ }^{2}$, Svitlana Lytvynova ${ }^{1}$, \\ Nadiia Pasko ${ }^{3}$, Svitlana Dubovyk ${ }^{3}$, Olena Orliyk ${ }^{4}$, Olga Siryk ${ }^{5}$, \\ and Vasyl Kyzenko ${ }^{6}$ \\ ${ }^{1}$ Institute of Information Technologies and Learning Tools of National \\ Academy of Educational Sciences of Ukraine, \\ 9 M. Berlyns'koho St., 04060 Kiev, Ukraine \\ ayb@iitlt.gov.ua, s.h.lytvynova@gmail.com \\ ${ }^{2}$ Sumy State University, Sumy, Ukraine \\ 3 Sumy National Agrarian University, Sumy, Ukraine \\ ${ }^{4}$ Scientific Research Institute of Intellectual Property, Kiev, Ukraine \\ 5 Kyiv National University named Taras Shevchenko, Kiev, Ukraine \\ ${ }^{6}$ Institute of Pedagogy of the National Academy of Educational Sciences \\ of Ukraine, Kiev, Ukraine
}

\begin{abstract}
This paper describes experimental study of cognitive performance degradation in high school students as the response to the psychophysiological changes in their activity support. The technique of studying the stability of cognitive abilities of high school students has revealed significant fluctuations in the speed and reliability of simple cognitive test tasks. A strong correlation between subjects' cognitive test activity and individual properties of their cardiovascular and nervous system, as well as energy regulation and solar physiological parameters (speed and density of solar wind) has been revealed $(\mathrm{R}=0.88 \ldots 0.91, \mathrm{p}<0.01)$. It is articulated that identified features of cognitive activity require further investigation and clarification of the mechanisms of regulation of such activity.
\end{abstract}

Keywords: Cognitive activity $\cdot$ Physiological support $\cdot$ External factors

\section{Introduction}

The influence of external and internal factors on an operator performance is known and well described in articles and patents [1]. But significance and role of certain physiological systems of mental performance support in relation to young people are less studied, namely which of them change their parameters more when cognitive performance degrades, especially in conditions of learning/training [2]. In addition, it is possible to expect that such changes can be more acute in adolescents because of their influence at learner performance and health [3]. Professional development of teachers should foresee this side of teaching [4], first of all, in digital learning environment [5], accounting systemic-structural concept to understand peculiar properties of learning 
activity [6] and as a systemic activity, in general [7], including nowadays' requirement of learning in lifespan [8].

Purpose. To study cognitive performance degradation in high school students as the response to the psychophysiological changes in their activity support.

\section{Method}

The research method was provided as the development of the method used in our previous research of the psychophysiological maintenance of operator cognitive performance [9] and further applications to learners as operator-researchers $[10,11]$. The last modification of the technique has been improved to develop adaptive learning systems [12].

1,5 months' experimental research was based on the use of a computer system SPPR (system of psycho-physiological research) to monitor the cognitive activity of high school students. The survey includes test task performance ( 2 types of logical tasks, and test for functional mobility of nervous processes' assessment), blood pressure and heart rate before and after the test performance, as well as electropuncture diagnostics (EPD) by Nakatani [13] was conducted after the test session for each subject (the 9th and 10th grade students, 11 subjects, participated in research 2 or 3 times per week after the necessary training): 6 subjects ( $1^{\text {st }}$ experiment) participated in experiments with measurement of the ECG interbit cardiointervals during the test performance and calculation of the ECG spectrum, 5 subjects ( $2^{\text {nd }}$ group) participated in experiments with measurement of EPD and physiological indices described above.

The adjusted tests block included [12, p. 22]:

Short-term memory test T2, where a table with 12 random numbers from 11 to 99 was presented to a subject. The number of correctly reproduced numbers was considered as a result.

Self-assessment test T4. It's an abridged version of Health-Activity-Mood test. The subject was proposed to give a subjective assessment of his/her state in 7-point scale, giving answers on five pairs of questions-characteristics.

Numbers permutation test (combinatorial) in ascending order T6. The test material: a sequence of numbers (from 0 to 9 ) which were not repeated and placed in a random order; the task was to rearrange the numbers in ascending order in a few steps, on each one could only change 2 adjacent numbers. Time for the task performance was free (the next task appeared just after entering the answer). The time and accuracy of the task performance were measured.

Numbers permutation test (combinatorial) in ascending order T9. The test material: a sequence of numbers (from 0 to 9 ) which were not repeated and placed in a random order; the task was to rearrange the numbers in descending order in a few steps, on each one could only change 2 adjacent numbers. Time for the task performance was free (the next task appeared just after entering the answer). The time and accuracy of the task performance were measured. 
Functional mobility and strength of nervous processes' test T10. Average 10TT and minimal 10 minTT time to execute the test tasks, maximum rate $10 \mathrm{~s} . \mathrm{min}$ to execute the test, time to rich it $10 \mathrm{t} \_$min.

Functional mobility and strength of nervous processes' in conditions of interference test T11. The same indicators with corresponding index 11.

In all tests we registered time of each task performance in milliseconds, correct (expected) and really entered answers. Besides, we used a subjective state assessment of the examinees by means of the reduced variant of the test "General_state - Activity Mood" (GsAM), test T4, at the beginning and at the ending of the test session (the indices of mood mood, serviceability FfD, attention atten, anxiety anxiety) prior to the beginning (index " 0 ") estimated and upon finishing the tests performance.

As indices of physiological "cost" of activity and the human state, we registered a heart rate HR and blood pressure (systolic ADs, diastolic ADd) by means of the digital blood pressure monitor Model LD11. The indices HR, ADs and ADd we registered during 5 min prior to the tests beginning (index " 1 ") and 5 min after finishing " 2 ", relaxation). The ECG interbit cardiointervals were registered with the cardiomonitor "Solveig".

To check an influence of the external physical factors on the cognitive task performance the solar activity was studied as in the research [13]. The data on influence of solar activity on a human health and some physiological systems are known, however results of study of cognitive activity associating with heliophysic parameters are not known in the scientific literature to date. In our preliminary pilot researches the precise connection between effectiveness of operator activity and parameters of a solar wind (SW) was revealed. With the purpose to study this phenomenon we registered indices of proton component of a solar wind - velocity SWsp $(\mathrm{km} / \mathrm{s})$ and density SWden (proton $/ \mathrm{sm}^{3}$ ) on the data from Internet site NASA, as well as parameters of the geomagnetic field (GMF) - planetary index Ks, index of "equivalent amplitude" A, as well as Wolf number of the solar spots.

\section{Discussion of Results}

As it has been demonstrated in our previous research, the relationship between physiological indices of adolescent and external factors (solar wind and atmospheric parameters) was medium, but significant.

In our current research with the $1^{\text {st }}$ group of subjects we studied relationship of indices of cognitive tests' performance (test T6, rate and reliability) and physiological parameters (blood pressure, average values and spectrum of the heart rate: 3 frequency bands - very low frequency VLF, low frequency LF and high frequency HF).

The results of the cognitive tests' performance confirmed the fact of changing the performance of the tests (average time and reliability of the decision of the test tasks) during the month, and those changes were fluctuating, that can be explained by the influence of the training load, as well as the influence of around weekly rhythms. Besides, the analysis of combined influence of the external factors (solar wind speed and density of its proton component at the time of testing) and physiological maintenance has revealed their parameters high correlation with tests performance speed, and 
especially reliability, in cognitive tests after selection of three the most informative independent variable in according to the standard procedure (standard package STATISTICA 6.0, a stepwise regression analysis).

An important result is not only a high correlation between the indicators of cognitive activity and other factors, but finding the most informative common factors among physiological ones for both task performance rate and reliability. They were as follows: SW density, $\mathrm{HF}, \mathrm{LF} / \mathrm{HF}$ and $\mathrm{HR} 1$. Besides, relationship was very high $\mathrm{R}=0.81 \ldots 0.98(\mathrm{p}<0.05)$.

In the $2^{\text {nd }}$ experiment EPD indices (only upper, measured on hands) were included into measurement of physiological parameters, as well as indices of the nervous system mobility ones.

The results of measurements demonstrated that partial coefficients of correlation are not very high (not higher than 0.5 ) for both rate of test performance and reliability in both cognitive tests T6 and T9 (Fig. 1).
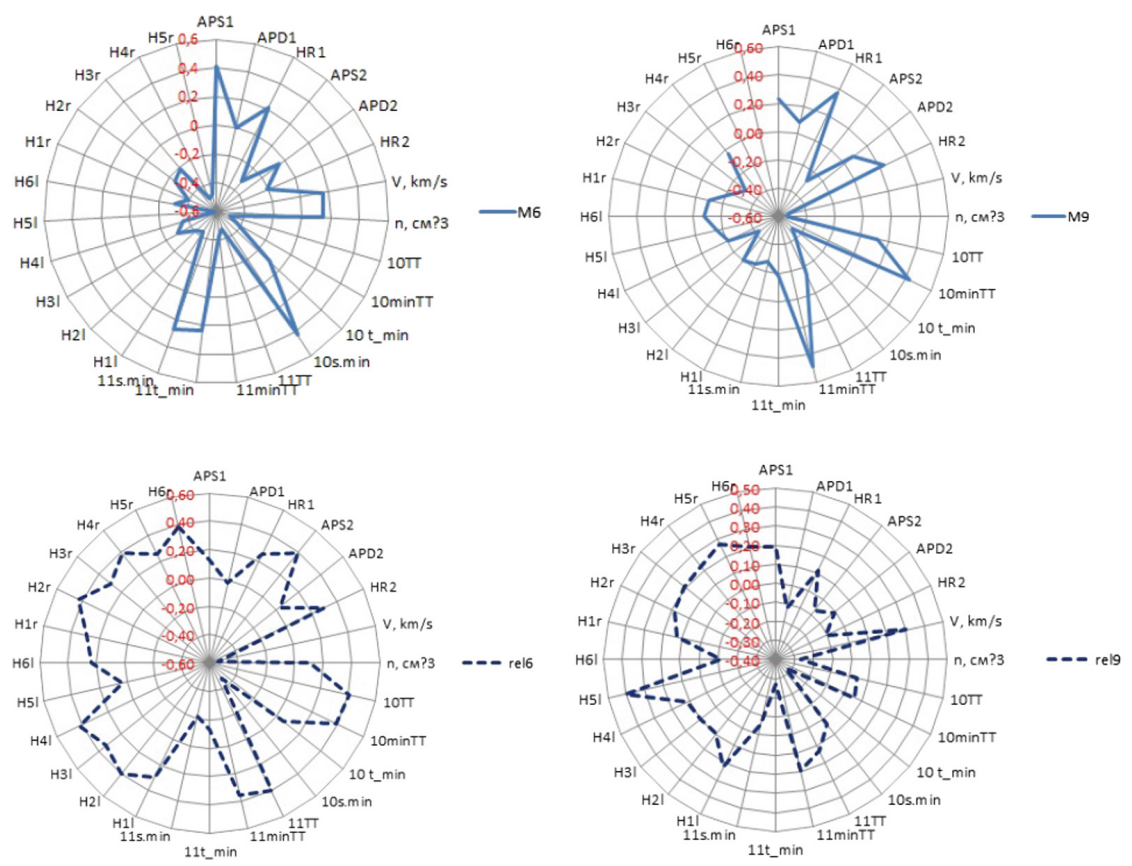

Fig. 1. Correlation histograms for average time (M6 and M9) and reliability (rel6 and re19) of test performance T6 and T9

It has been revealed that the composition of the factors varies (the individuality of people affects) for different tests and subjects, but the indices of the nervous system features, energy balance/imbalance and indices of the solar wind were substantially influenced, as well as some subjective indices of mood and rest. 
Analysis of the effects of energy balance (by Nakatany), autonomic regulation (on the parameters of the heart rate and blood pressure), the features of the nervous system (in particular, functional mobility of the nervous processes) and external factors (solar wind speed and density of its proton component at the time of testing) has revealed their high correlation with tests performance speed, and especially reliability, in cognitive tests after selection of three the most informative independent variable.

An important result is not only a high correlation between the indicators of cognitive activity and other factors, but finding the most informative factors among physiological ones. It has been revealed that the composition of the factors varies (the individuality of people affects) for different tests and subjects, but the indices of the nervous system features, energy balance/imbalance and indices of the solar wind were substantially influenced, as well as some subjective indices of mood and rest. At the same time, heart rate and blood pressure were not significant.

By selecting the 3 most informative independent variables according to the standard procedure, a stepwise regression analysis revealed: the coefficient of multiple correlation of the test performance rate (index corresponds to the test number) $R 6_{M}=0.7 \ldots$ $0.93(\mathrm{p}<0.01), \mathrm{R} 9 \mathrm{M}=0.95 \ldots 0.97(\mathrm{p}<0.001)$; multiple correlation coefficient of test performance, $\mathrm{R} 6_{\text {rel }}=0.88 \ldots 0.91(\mathrm{p}<0.01), \mathrm{R} 9_{\text {rel }}=0.95 \ldots 0.97(\mathrm{p}<0.01)$. In other words, the use of extended physiological indicators in the $2^{\text {nd }}$ experiment allowed to achieve very high accuracy of assessment of the rate and reliability of cognitive test performance.

\section{Conclusion}

The technique of studying the stability of cognitive abilities of high school students has revealed significant fluctuations in the speed and reliability of simple cognitive test tasks.

A strong correlation between subjects' cognitive test activity and individual properties of their cardiovascular and nervous system, as well as energy regulation and solar physiological parameters (speed and density of solar wind) has been revealed $(\mathrm{R}=0.88 \ldots 0.91, \mathrm{p}<0.01)$.

Identified features of cognitive activity require further investigation and clarification of the mechanisms of regulation of such activity.

Acknowledgments. This research has been supported by the Institute of Information Technologies of the National Academy of Pedagogic Science.

\section{References}

1. Burov, O.: Day-to-day monitoring of an operator's functional state and fitness-for-work: a psychophysiological and engineering approach. In: Chebykin, O.Y., Bedny, G., Karwowski, W. (eds.) Ergonomics and Psychology: Developments in Theory and Practice, pp. 107-126. CRC Press, Boca Raton (2008) 
2. Zubchenko, T.M., Naumenko, Yu.A., Burov, O.Yu.: ICT for studying the dynamics of school abilities under the influence of external and internal factors. Komp'iuter u shkoli ta sim'ii 1, 3-14 (2017)

3. McLachlan, S., Dube, K., Kyrimi, E.: On behalf of HiKER (Health Informatics and Knowledge Engineering Research) Group: LAGOS: learning health systems and how they can integrate with patient care. BMJ Health Care Inform. (2019). https://doi.org/10.1136/ bmjhci-2019-100037

4. Lytvynova, S., Melnyk, O.: Professional development of teachers using cloud services during non-formal education. In: Proceedings of the 12th International Conference on ICTERI 2016, Kyiv, Ukraine, 21-24 June 2016. Integration, Harmonization and Knowledge Transfer, vol. 1614, pp. 648-655. CEUR-WS.org (2016). http://ceur-ws.org/Vol-1614/paper_51.pdf

5. Michalak, D., Rozmus, M.: Methods and tools for acquiring high-quality skills in digital era - innovative practices and results from 3DSPEC and e-MOTIVE projects. In: Karwowski, W., et al. (eds.) AHFE 2019. AISC, vol. 963, pp. 260-270 (2019). https://doi.org/10.1007/ 978-3-030-20135-7_26

6. Bedny, G.Z., Karwowski, W.: A systemic-structural activity approach to the design of human-computer interaction tasks. Int. J. Hum.-Comput. Interact. 16(2), 235-260 (2003)

7. Pinchuk, O., Burov, O., Lytvynova, S.: Learning as a systemic activity. In: Karwowski, W., et al. (eds.) AHFE 2019. AISC, vol. 963, pp. 335-342 (2019). https://doi.org/10.1007/978-3030-20135-7_26

8. Burov, O.: Life-long learning: individual abilities versus environment and means. In: Proceedings of the 12th International Conference on ICTERI 2016, Kyiv, Ukraine, 21-24 June 2016. Integration, Harmonization and Knowledge Transfer, vol. 1614, pp. 608-619. CEUR-WS.org (2016). http://ceur-ws.org/Vol-1614/paper_86.pdf

9. Burov, A.: Evaluation of functional state of operators on parameters of mental serviceability. Hum. Physiol. 2, 29-36 (1986)

10. Lavrov, E., Lavrova, O.: Intelligent adaptation method for human-machine interaction in modular E-learning systems. In: Proceedings of the 15th International Conference on ICT in Education, Research and Industrial Applications Integration, Harmonization and Knowledge Transfer. Volume II: Workshops, Kherson, Ukraine, 12-15 June 2019, pp. 1000-1010 (2019)

11. Mulder, L.J.M., Van Roon, A., Veldman, H., Laumann, K., Burov, A., Quispel, L., Hoogeboom, P.J.: How to use cardiovascular state changes in adaptive automation. In: Hockey, G.R.J., Gaillard, A.W.K., Burov, O. (eds.) Operator Functional State. The Assessment and Prediction of Human Performance Degradation in Complex Tasks. NATO Science Series, pp. 260-272. IOS Press, Amsterdam (2004)

12. Burov, O.Yu., Pinchuk, O.P., Pertsev, M.A., Vasylchenko, Y.V.: Use of learners' state indices for design of adaptive learning systems. Inf. Technol. Learn. Tools 68(6), 20-32 (2018)

13. Rudenko, S.A.: Study of school children chronic fatigue with method of acupuncture diagnostic by Nakatani. In: Seminar Proceedings Psychophysiological Aspects of Giftedness: Theory and Practice, Kyiv, Ukraine, 3 February 2012, pp. 92-97 (2012) 